\title{
Evaluation of Garlic-Lemon Therapy on Clinical and Angiographic Outcomes in Patients with High Risk Acute Coronary Syndrome \\ E Yeter ${ }^{1}$, H Sunman $^{1 *}$, M Aytürk $^{2}$, M Erat $^{1}$, E Algül ${ }^{1}$, HE Pamukçu $^{1}$, LD Asarcıkl1 ${ }^{1}$, M Bilgin $^{1}, \mathrm{~T}$ Çimen ${ }^{1}$, A Akyel $^{1}$, S Açıkel ${ }^{1}, \mathrm{M}_{\text {Doğan }}^{1}$
}

\begin{abstract}
Objective: Several natural supplementations have been investigated over centuries for a wide variety of ailments. There is limited information available on the effect and safety of these natural supplementations on patients with high risk coronary artery disease. We aim to evaluate the results of garlic and lemon therapy via clinical and angiographic outcomes in patients with ST-elevation myocardial infarction.
\end{abstract}

Methods: Patients who had $>70 \%$ non-culprit coronary stenosis were included in the study. Patients who underwent optimal medical therapy were randomized to the treatment. At the end of the followup, patients were evaluated in terms of clinical and angiographic outcomes.

Results: Of the 77 subjects enrolled in the study, fifty-eight patients (28 from the treatment group and 30 from the control group) completed the study period successfully. During the follow-up period, the need for early revascularization appeared later in the treatment group as compared to the control group. According to the Rose angina survey, the rates of definite angina, and probable angina were lower in the treatment group compared to the control group. The mean stenosis, in the treatment group, at the baseline and that in the $3^{\text {rd }}$ month was $49.32 \pm 20.45$ and $48.48 \pm 20.64$, respectively $(p=$ $0.168)$; whereas, in the control group, it was $49.93 \pm 22.71$ and $49.14 \pm 22.63$, respectively $(\mathrm{p}=0.116)$.

Conclusion: Based on the present study, no significant change was observed in atherosclerotic plaques at the end of the three-month treatment period, although it resulted in symptomatic benefits. However, the absence of serious adverse events within the 3-month period is important as it suggests the safety of the treatment.

Keywords: Acute coronary syndrome, garlic-lemon therapy, natural supplementation

From: ${ }^{1}$ Department of Cardiology, Ministry Of Health Dışkap1 Yıldırım Beyazıt Research and Educational Hospital, Ankara, Turkey. ${ }^{2}$ Department of Cardiology, Keçiören Training and Research Hospital, Ankara, Turkey.

Correspondence Dr H Sunman, Department of Cardiology, Ministry of Health, Dışkapı Yıldırım Beyazıt Education and Research Hospital, 06110, Ankara, Turkey. E-mail: hamzasunman@gmail.com 


\section{INTRODUCTION}

Coronary artery disease (CAD) due to atherosclerosis is one of the major causes of mortality and morbidity at a global level. The importance of CAD is gradually increasing day by day due to its high prevalence in the population and the consequent socioeconomic burden because it ultimately leads to myocardial infarction, heart failure, sudden cardiac death, and related health problems. Nevertheless, CAD-related morbidity and mortality have significantly decreased in the past decades owing to the scientific and technological advancements in the treatment of the disease. Major advancements have been made in the treatment of CAD with the invention of new drugs and with the increased number of revascularization methods. However, pharmacological, interventional, and surgical methods might not be available universally for all the patients or each patient might not benefit in the same way as the others. In addition, these therapeutic methods are not affordable due their high cost. While various drugs such as antiaggregants, anticoagulants or statins have been investigated, studies on natural supplementations are gradually increasing. Better side effect profiles, lower cost, and increasing number of investigations encourage new studies on this subject $(1,2)$.

Several natural supplementations have been investigated for centuries for a wide variety of ailments. Lemon, which is one among these, is being widely used in some countries for the treatment of hypertension (3). In addition, garlic has been used as both food and medicine for thousands of years, and there were some scientific support regarding its property to suppress the development of atherosclerosis (4). Studies on animals suggest that garlic exerts a hypocholesterolemic effect and causes a reduction in the area of atherosclerotic plaque $(5,6)$. Garlic and lemon in combination have been valued in traditional and folk medicine in some cultures to remedy cardiovascular disease over centuries; however, its role in prevention or regression of atherosclerosis in patients with CAD has still not been 
investigated. In addition, studies on natural supplementations including garlic have been mainly conducted in stable CAD. There are limited data available on the effect and safety of such natural supplementations in high risk CAD. The present study aims to investigate clinical and angiographic outcomes of traditional garlic-lemon therapy in patients with high risk acute coronary syndrome (ACS).

\section{MATERIALS AND METHODS}

\section{Study design and selection of subjects}

The patients, admitted to our clinic for ST-elevation myocardial infarction (STEMI) and underwent primary percutaneous coronary intervention (PCI), were evaluated for the study. The enrolled patients were having $70-90 \%$ stenosis in the coronary arteries other than those undergoing primary PCI. All patients were receiving optimal medical treatment. The patients in the treatment group were randomized to receive garlic-lemon therapy as compared to the control group. The patients were randomized considering gender, localization of MI, and left ventricle systolic function into account. Exclusion criteria included conditions such as cardiogenic shock at presentation, resistant angina, and ventricular arrhythmia, which require revascularization of non-culprit vessel at the same session as well as the patients with right ventricular involvement and with left main coronary artery stenosis. In addition, patients who were allergic to garlic or lemon were also excluded. All subjects were informed, and a written consent was obtained. The study was approved by the local Ethics Committee.

\section{Baseline definitions and assessment of risk factor}

The demographics, medical histories, and medication usage were recorded. Hypertension was defined as a diastolic blood pressure of $\geq 90 \mathrm{mmHg}$ or a systolic blood pressure of $\geq 140$ mmHg or the self-reported use of antihypertensive $\operatorname{drug}(\mathrm{s})$ [6]. Diabetes mellitus was 
considered present in patients with a history of oral anti-diabetic drug or insulin use or fasting blood glucose of $\geq 126 \mathrm{mg} / \mathrm{dL}$ at the beginning of the study. A history of cigarette smoking was considered present if the subject was a former or current smoker. A positive family history was defined as the presence of CAD in a parent or sibling under the age of 55 years for men and 65 years for women.

\section{Natural supplementation}

Natural supplement was prepared in accordance with traditional usage by adding $30 \mathrm{~g}$ of crushed garlic into $2.5 \mathrm{~L}$ of lemon juice and storing in a closed container for 6 weeks avoiding exposure to light. Thereafter, this supplementation was administered orally at a dose of $40 \mathrm{~mL}$ (approximately $2 \times 500 \mathrm{mg}$ garlic) twice daily. The mixture was given to the patients together with the monthly schedule of administration, and the treatment continued for three months.

\section{Follow-up}

The patients were followed-up through visits to the clinic on the first week, and the first and second month; while angiography was performed on the third month. Early coronary angiography was performed for ongoing Canadian Cardiovascular Society class (CCS) III symptoms (despite antianginal medications at maximum tolerated doses), or if the patient had been admitted with further ACS. ACS was defined based on the criteria of chest pain, elevated serum cardiac biomarker levels, and typical alterations of the ECG including ST segment deviations and new left bundle branch block; unstable angina was defined as the clinical presentation of ACS without cardiac biomarker elevation including worsening angina requiring hospitalization despite maximal medical therapy. At the end of the follow-up, chest pain was evaluated through the Rose Angina Questionnaire. Patients were classified into one of the four groups: definite angina, possible angina, non-exertional chest pain or no chest pain. Definite angina according to standard criteria is chest pain or discomfort which: (1) was started by exertion; (2) was situated in the central or left anterior chest; (3) forced the subject 
to slow down or stop; (4) was relieved if the subject did so; and (5) was relieved within 10 min. Possible angina was defined as chest pain started by exertion, but not fulfilling all of the four additional criteria for definite angina. Chest pain that was not started by exercise was classified as non-exertional chest pain (7). Laboratory measurements included assessments of complete blood count, liver and kidney function, plasma total cholesterol, low-density lipoprotein cholesterol (LDL-C), high-density lipoprotein cholesterol (HDL-C), and triglycerides.

\section{Angiographic evaluation}

Control angiography images obtained at the end of third month were compared with baseline angiography images. The coronary segments were reviewed according to the modified 16segment American Heart Association classification system, including the left main coronary artery; proximal, mid, and distal left anterior descending artery; proximal, mid, and distal diagonal/intermediate branch; proximal, mid, and distal circumflex artery; proximal, mid, and distal obtuse marginal branch; and proximal, mid, and distal right coronary artery. However, coronary artery segments with a diameter of less than $2 \mathrm{~mm}$ and those that underwent revascularization during primary PCI were not included in evaluation. Potential coronary spasm was eliminated by administering nitrate prior to both angiographic imaging procedures. Angiography obtained at angles that displayed the best image of the lesions was used during both the analyses. Stenosis of coronary segments were visually evaluated double-blindly by two competent interventional cardiologists. The analysis was performed by calculating the arithmetical mean of the results.

\section{Statistical analysis}

The continuous variables were expressed as mean \pm standard deviation, and categorical variables were expressed as absolute numbers and percentages. The categorical variables were analyzed by Chi-square-test. Comparisons of the continuous variables between the two groups 
were performed using the independent samples t-test. The differences between the two groups were evaluated using adjusted Chi-square test for categorical variables and two-tailed student t-test or Man-Whitney test for continuous variables. Interobserver agreements were measured with intraclass correlation coefficients (ICC). Statistical analyses were performed using SPSS statistical software. A p-value of $<0.05$ was considered statistically significant.

\section{RESULTS}

\section{Patients' characteristics}

Of the 77 subjects enrolled in the study, 38 were randomized to the treatment group and 39 to the control. In treatment group, six patients withdrew their consent after early days of the study on their own accord as they did not want to receive treatment. Twenty eight patients in the treatment group and thirty patients in the control group completed the follow-up period successfully. The mean age of the patients those were included in the study was $58.17 \pm 10.6$ years, and $20(34.5 \%)$ patients were female. Of the patients that completed the study, 20 (34.5\%) were suffering from hypertension and 14 (24.1\%) from diabetes. In addition, both the groups were statistically similar in terms of baseline LDL-C, HDL-C, triglycerides, ejection fraction, systolic/diastolic blood pressure, and TIMI scores. Acetylsalicylic acid, clopidogrel, $\beta$-blocker, ACE inhibitor/ARB, and statins (atorvastatin) were prescribed to nearly all of the study patients. Baseline characteristics are presented in Table 1.

\section{Follow-up data and angiographic findings}

Early revascularization before the third month was performed in 13 of 71 patients enrolled in the study. Early revascularization was performed in four patients (1 unstable angina pectoris and 3 CCS class-3 angina) in the treatment group and in nine patients ( 1 non-ST elevation 
myocardial infarction, 2 unstable angina pectoris, and 6 CCS-3 angina) in the control group ( $\mathrm{p}$ $=0.252$ ). The necessity for early revascularization during follow-up period appeared later in the treatment group as compared to the control group (mean $24.0 \pm 9.4$ vs. $43.5 \pm 12.5$ days, $\mathrm{p}=$ 0.005). PCI was performed in all of 13 patients those underwent early revascularization excluding one, who underwent coronary bypass surgery. Based on angina survey performed at the end of the third month in 58 patients those completed follow-up period, definite angina, probable angina, and non-exertional angina were less prevalent in the treatment group compared to the control group [2 (7.1\%) vs. $6(20.0 \%), 5(17.9 \%)$ vs. $12(40.0 \%), 13(46.4 \%)$ vs. $5(16.7 \%)$, and $8(28.6 \%)$ vs. $7(23.3 \%)$, respectively; p $=0.038]$. All the patients were receiving $\beta$-blocker after primary PCI. The necessity for additional anti-anginal drug during follow-up period appeared in $5(17.9 \%)$ patients in the treatment group and in $7(23.3 \%)$ in the control group $(\mathrm{p}=0.600)$. In addition, 58 patients were evaluated via coronary angiography at the end of the follow-up period. Instant serious restenosis was detected in 2 (7.1\%) patients in the treatment group and in $3(10 \%)$ patients in the control group. All patients, except for three in the treatment group and two in the control group, underwent PCI during third-month angiography. After removing the coronary artery segments, which underwent intervention before being included in the study, baseline and control angiographic images of 580 coronary segments were examined by two competent interventional cardiologists. There was a good agreement between the two observers $[\mathrm{ICC}=0.792(95 \% \mathrm{CI}$ : $0.755-0.823)$ for the first angiography and ICC $=0.780$ (95\% CI: $0.741-0.813)$ for the control]. Changes were observed in 25 (8.7\%) segments (16 regressions and 9 progressions) in the treatment group and in $30(10.2 \%)$ segments (17 regressions and 13 progressions) in the control group $(\mathrm{p}=0.323)$. While baseline and three-month mean stenosis values in all segments except for those who underwent primary PCI were $16.84 \pm 20.99$ and $16.82 \pm 20.71$, respectively in the treatment group ( $\mathrm{p}=0.918$ ); the values were $18.47 \pm 22.48$ and 18.44 
\pm 22.14 , respectively, in the control group $(\mathrm{p}=0.830)$. The baseline and three-month mean stenosis values of the segments with stenosis over $20 \%$ were $49.32 \pm 20.45$ and $48.48 \pm 20.64$, respectively, in the treatment group $(\mathrm{p}=0.168)$, whereas $49.93 \pm 22.71$ and $49.14 \pm 22.63$, respectively in the control group $(\mathrm{p}=0.116)$. The laboratory analyses performed at the end of three months revealed significant decrease in LDL, TG, and blood pressure values in both the groups. However, the decrease in the LDL, TG, and blood pressure values at the third month versus baseline was not statistically significantly different between the treatment and control groups (LDL: $63.1 \pm 17.8$ vs. $62.6 \pm 22.3, \mathrm{p}=0.925$; TG: $40 \pm 69.3$ vs. $41 \pm 69.2, \mathrm{p}=0.416$; systolic blood pressure: $15.8 \pm 7.4$ vs. $17.0 \pm 9.9, \mathrm{p}=0.614$; diastolic blood pressure: $10.6 \pm 7.5$ vs. $12.5 \pm 4.1, \mathrm{p}=0.244)$

\section{Tolerability and acceptability}

Most of the participants found taking the combination therapy easy and acceptable. However, six patients did not want to receive treatment at the start of the study. Two patients in the treatment group experienced nausea at the beginning of treatment, but improved during the following days, and they completed the treatment period successfully. The most important issue of discomfort, even if mild, was bad breath. Over the course of treatment period, none of either of liver toxicity, acute renal insufficiency, symptomatic hypotension or major bleeding was reported in the treatment group or in the control group. 


\section{DISCUSSION}

The present study demonstrates that garlic-lemon therapy, as a natural supplementation, reduces anginal symptoms and delays the need for revascularization in high-risk ACS patients. With regard to the atherosclerotic stenosis, no statistically significant regression was observed at the end of three months as compared to the control group. Nevertheless, the present study, which has been conducted in high-risk ACS patients, ascertains the safety of this therapy.

Previous studies have reported that garlic reduces total serum cholesterol both by inhibiting LDL cholesterol synthesis and by increasing serum HDL cholesterol. The studies performed in laboratory mice reported that blood and tissue samples of the mice, the diets of which have been supplemented with garlic, contain lower amount of lipid and, in addition, they have lower hepatic cholesterol and triglyceride levels. The studies in rabbits demonstrated that preexisting atherosclerotic deposition could be reversed by regular garlic consumption (5). Some studies demonstrated that garlic consumption might reduce systolic and diastolic blood pressure (8). Favorable effect of garlic therapy on atherosclerosis has been demonstrated in the studies that considered the measurements of carotid intima media thickness and coronary calcium score $(2,9)$. Garlic contains amino acids such as cysteine, glutamine, isoleucine, and methionine, which protects the cells against harmful effects of free radicals. It reduces the oxidation of LDL-C, in particular, by increasing the levels of endogenous antioxidants. In addition, platelet aggregation and subsequent thrombus formation are significantly reduced by garlic. The mechanism of inhibition of platelet aggregation is thought to function via the inhibition of calcium mobilization. Fibrinolysis is also enhanced by garlic, resulting in dissolution of clots and thrombi. In vitro studies have demonstrated that aged garlic extract improves circulation and blood properties by preventing lipid peroxidation and hemolysis in oxidized erythrocytes. It has been demonstrated that garlic therapy reduces 
endothelial injury and smooth muscle cell migration. It was determined that garlic therapy reduces plaque progression by influencing homocysteine synthesis (10-13). Besides garlic, several investigations have demonstrated that consuming citrus juice decreased endothelial dysfunction, reduced inflammation, and attenuates vascular remodeling partly through the reduction of oxidative stress (14-16). Citrus juice is also widely used to prevent sudden increase in blood pressure (3). In the present study, lower angina score at the end of the treatment as compared to the control group and a delay in the need for PCI in non-culprit vessel might be due to these effects of garlic-lemon therapy.

The effects of natural supplementations on atherosclerosis have been an important area of research since a long time. The effects of garlic alone or in combination with various supplements (coenzyme Q10, B vitamins, folic acid, L-arginine, etc.) have been investigated. The present study investigated the combination of garlic with lemon juice. Based on the observations, it was recognized that the combined use of lemon juice and garlic is quite common. Several reasons for preferring this combination have been identified. The mixture of lemon juice with crushed garlic is better tolerated by the subjects. Smell and irritation are less common. The fact that the mixture can be prepared, stored, and consumed orally facilitates adherence to the treatment regime. In addition, both the components of the mixture viz. garlic and lemon juice have been known to exert a favorable effect on blood pressure. In the present study, changes in lipid profile at the end of three months showed no difference between the groups. Aggressive high-dose statin therapy in ACS patients might have masked this benefit. The present study determined no difference between the treatment and control groups in terms of decrease in blood pressure levels. The fact that almost all of the patients have been receiving $\beta$-blocker and $\mathrm{ACEI} / \mathrm{ARB}$ therapy might have masked the effect of garlic-lemon therapy on blood pressure. 
In addition to its efficacy against CADs, tolerability was found to be quite good in the present study, which has been conducted in high risk patients. No significant adverse event other than gastrointestinal symptoms in two patients (nausea, dyspepsia, etc.) was encountered. Three patients quit the study at the beginning of the study without giving any reason. At the conclusion of the treatment, approximately one third of the patients in the treatment group stated that they will continue the treatment at home as they feel comfortable although they had been told that stenosis in their vessels showed no regression angiographically. This result suggests that there may be microvascular improvements despite the absence of macrovascular benefits.

This study has some of its own limitations. First, the period of treatment with lemongarlic was relatively short, and the atherosclerotic changes were measured after three months of supplementation. It might be possible that longer period of treatment and later evaluation may better document atherosclerotic changes. Second, the patients were receiving medications that may potentially influence plasma lipid profiles, blood pressure, and platelet functions. Nevertheless, the observance of similar effects in the control group helps us to understand the effect of natural supplementations. Third, atherosclerotic plaques have been evaluated visually. Fourth, anti-oxidant and anti-inflammatory effects have not been investigated. Finally, the exclusion of placebo in the control group is also one of the limitations of the study.

\section{CONCLUSION}

The present study investigated the effects of natural supplementations in high-risk ACS patients; wherein a combination of garlic-lemon juice offered symptomatic benefits. Although no angiographic regression was observed in atherosclerotic plaques, it was found to be 
associated with a decrease in the need for early vascularization. Moreover, the absence of serious adverse events over the course of three months of the natural supplementation is another important result of the study.

\section{ACKNOWLEDGEMENT}

On behalf of all of authors of the manuscript, I would like to express our sincere appreciation to all nurses and staff of our clinic who have helped to make the study, especially Yurdagül Kortun, Melek Bakar, and Esma Demirkol.

\section{AUTHORS' NOTE}

There is no potential conflict of interests or financial activities related to the present paper to disclose. 


\section{REFERENCES}

1. Karaarslan S, Alihanoglu YI, Yildiz BS, Sonmez O, Soylu A, Bacaksiz A, et al. Appropriateness of the current guidelines on reperfusion treatment for patients applying to our hospital with ST-segment elevation acute myocardial infarction. Turk Kardiyol Dern Ars 2012; 40: 493-8.

2. Mahdavi-Roshan M, Zahedmehr A, Mohammad-Zadeh A, Sanati HR, Shakerian F, Firouzi A, et al. Effect of garlic powder tablet on carotid intima-media thickness in patients with coronary artery disease: A preliminary randomized controlled trial. Nutr Health 2013; 22: 143-55.

3. Adibelli Z, Dilek M, Akpolat T. Lemon juice as an alternative therapy in hypertension in Turkey. Int J Cardiol 2009; 135: e58-9.

4. Prasad K, Mantha SV, Kalra J, Lee P. Prevention of Hypercholesterolemic Atherosclerosis by Garlic, an Antixoidant. J Cardiovasc Pharmacol Ther 1997; 2: 30920.

5. Durak I, Ozturk HS, Olcay E, Guven C. Effects of garlic extract supplementation on blood lipid and antioxidant parameters and atherosclerotic plaque formation process in cholesterol-fed rabbits. J Herb Pharmacother 2002; 2: 19-32.

6. Petrovska BB, Cekovska S. Extracts from the history and medical properties of garlic. Pharmacogn Rev 2010; 4: 106-10.

7. Lampe FC, Whincup PH, Wannamethee SG, Ebrahim S, Walker M, Shaper AG. Chest pain on questionnaire and prediction of major ischaemic heart disease events in men. Eur Heart J 1998; 19: 63-73.

8. Steiner M, Khan AH, Holbert D, Lin RI. A double-blind crossover study in moderately hypercholesterolemic men that compared the effect of aged garlic extract and placebo administration on blood lipids. Am J Clin Nutr 1996; 64: 866-70. 
9. Zeb I, Ahmadi N, Nasir K, Kadakia J, Larijani VN, Flores F, et al. Aged garlic extract and coenzyme Q10 have favorable effect on inflammatory markers and coronary atherosclerosis progression: A randomized clinical trial. J Cardiovasc Dis Res 2012; 3: 185-90.

10. Moriguchi $\mathrm{T}$, Takasugi N, Itakura $\mathrm{Y}$. The effects of aged garlic extract on lipid peroxidation and the deformability of erythrocytes. J Nutr 2001; 131: 1016S-9S.

11. Budoff MJ, Ahmadi N, Gul KM, Liu ST, Flores FR, Tiano J, et al. Aged garlic extract supplemented with B vitamins, folic acid and L-arginine retards the progression of subclinical atherosclerosis: a randomized clinical trial. Prev Med 2009; 49: 101-7.

12. Rahman K, Lowe GM. Garlic and cardiovascular disease: a critical review. J Nutr 2006; 136: 736S-40S.

13. Larijani VN, Ahmadi N, Zeb I, Khan F, Flores F, Budoff M. Beneficial effects of aged garlic extract and coenzyme Q10 on vascular elasticity and endothelial function: the FAITH randomized clinical trial. Nutrition 2013; 29: 71-5.

14. Ohnishi A, Asayama R, Mogi M, Nakaoka H, Kan-No H, Tsukuda K, et al. Drinking citrus fruit juice inhibits vascular remodeling in cuff-induced vascular injury mouse model. PLoS One 2015; 10: e0117616.

15. Deyhim F, Lopez E, Gonzalez J, Garcia M, Patil BS. Citrus juice modulates antioxidant enzymes and lipid profiles in orchidectomized rats. J Med Food 2006; 9: $422-6$.

16. Buscemi S, Rosafio G, Arcoleo G, Mattina A, Canino B, Montana M, et al. Effects of red orange juice intake on endothelial function and inflammatory markers in adult subjects with increased cardiovascular risk. Am J Clin Nutr 2012; 95: 1089-95. 
Table 1: Baseline characteristics of the patients

\begin{tabular}{|c|c|c|c|}
\hline Parameters & $\begin{array}{l}\text { Treatment group } \\
(\mathrm{n}=28)\end{array}$ & $\begin{array}{l}\text { Control group } \\
(\mathrm{n}=30)\end{array}$ & p-value \\
\hline Age (years) & $59.11 \pm 10.3$ & $57.30 \pm 11.0$ & 0.522 \\
\hline Gender (\% female) & $9(32.1)$ & $11(36.7)$ & 0.717 \\
\hline BMI $\left(\mathrm{kg} / \mathrm{m}^{2}\right)$ & $26.3 \pm 2.5$ & $26.1 \pm 1.5$ & 0.734 \\
\hline Hypertension (n, \%) & $17(60.7)$ & $19(63.3)$ & 0.837 \\
\hline Diabetes $(n, \%)$ & $6(21.4)$ & $8(26.7)$ & 0.641 \\
\hline History of smoking (n, \%) & $13(46.4)$ & $21(70.0)$ & 0.069 \\
\hline Family history (n, \%) & $11(39.3)$ & $19(63.3)$ & 0.067 \\
\hline Systolic BP (mmHg) & $137.8 \pm 15.5$ & $142.7 \pm 13.3$ & 0.202 \\
\hline Diastolic BP (mmHg) & $77.1 \pm 11.4$ & $77.5 \pm 13.8$ & 0.898 \\
\hline Ejection Fraction (\%) & $48.3 \pm 8.7$ & $45.9 \pm 5.7$ & 0.215 \\
\hline Medications (n, \%) & & & $>0.500$ \\
\hline Aspirin & $32(100)$ & $39(100)$ & \\
\hline Clopidogrel & $32(100)$ & $39(100)$ & \\
\hline B-blocker & $32(100)$ & $37(94.9)$ & \\
\hline ACEi/ARB & $32(100)$ & $37(94.9)$ & \\
\hline Statin & $32(100)$ & $39(100)$ & \\
\hline LDL (mg/dl) & $140.7 \pm 22.9$ & $140.1 \pm 24.9$ & 0.931 \\
\hline HDL(mg/dl) & $38.6 \pm 6.5$ & $41.4 \pm 8.2$ & 0.070 \\
\hline TG (mg/dl) & $231 \pm 87$ & $230 \pm 53$ & 0.940 \\
\hline Localization of MI (n, \%) & & & 0.974 \\
\hline Anterior & $5(17.9)$ & $7(23.3)$ & \\
\hline Anterolateral & $4(14.3)$ & $3(10.0)$ & \\
\hline Inferior & $12(42.9)$ & $12(40.0)$ & \\
\hline Inferolateral & $5(17.9)$ & $6(20.0)$ & \\
\hline Inferoposterior & $2(7.1)$ & $2(6.7)$ & \\
\hline $\begin{array}{l}\text { TIMI score after primary PCI } \\
\text { (mean) }\end{array}$ & $2.7 \pm 0.3$ & $2.8 \pm 0.3$ & 0.838 \\
\hline
\end{tabular}

ACEi/ARB, angiotensin converting enzyme inhibitor/angiotensin receptor blocker; BP, blood pressure; HDL, high density lipoprotein; LDL, low density lipoprotein; MI, myocardial infarction; PCI, percutaneous coronary intervention; TG, triglyceride 
Table 2: Clinical, laboratory and angiographic results

\begin{tabular}{|c|c|c|c|c|c|c|c|}
\hline Parameters & \multicolumn{3}{|c|}{ Treatment group $(n=28)$} & \multicolumn{3}{|c|}{ Control group $(\mathrm{n}=30)$} & $\mathrm{p}$ \\
\hline $\begin{array}{l}\text { Chest pain (n, } \\
\%)\end{array}$ & & & & & & & $\begin{array}{l}0.03 \\
8\end{array}$ \\
\hline Definite angina & $2(7.1 \%)$ & & & $6(20.0 \%)$ & & & \\
\hline Possible angina & $5(17.9 \%)$ & & & $12(40.0 \%)$ & & & \\
\hline Non-exertional & $13(46.4 \%)$ & & & $5(16.7 \%)$ & & & \\
\hline $\begin{array}{l}\text { chest pain } \\
\text { No chest pain }\end{array}$ & $8(28.6 \%)$ & & & $7(23.3 \%)$ & & & \\
\hline $\begin{array}{l}\text { Additional } \\
\text { anti-anginal } \\
\text { drug usage (n, } \\
\% \text { ) }\end{array}$ & $5(17.9 \%)$ & & & $7(23.3 \%)$ & & & $\begin{array}{l}0.60 \\
0\end{array}$ \\
\hline $\begin{array}{l}\text { Early } \\
\text { revascularizati } \\
\text { on }(\mathbf{n}, \%)\end{array}$ & $4(14.2 \%)$ & & & $9(30.0 \%)$ & & & $\begin{array}{l}0.25 \\
2\end{array}$ \\
\hline $\begin{array}{l}\text { Early } \\
\text { revascularizati } \\
\text { on time (days) }\end{array}$ & $24.0 \pm 9.4$ & & & $43.5 \pm 12.5$ & & & $\begin{array}{l}0.00 \\
5\end{array}$ \\
\hline $\begin{array}{l}\text { In-stent } \\
\text { restenosis (n, } \\
\% \text { ) }\end{array}$ & $2(7.1 \%)$ & & & $3(10 \%)$ & & & $\begin{array}{l}0.72 \\
2\end{array}$ \\
\hline $\begin{array}{l}\text { Reduction in } \\
\text { LDL level } \\
\text { (mg/dl) }\end{array}$ & $63.1 \pm 17.8$ & & & $62.6 \pm 22.3$ & & & $\begin{array}{l}0.92 \\
5\end{array}$ \\
\hline $\begin{array}{l}\text { Reduction in } \\
\text { TG level } \\
\text { (mg/dl) }\end{array}$ & $40 \pm 69.3$ & & & $41 \pm 69.2$ & & & $\begin{array}{l}0.41 \\
6\end{array}$ \\
\hline $\begin{array}{l}\text { Reduction in } \\
\text { SBP (mmHg) }\end{array}$ & $15.8 \pm 7.4$ & & & $17.0 \pm 9.9$ & & & $\begin{array}{l}0.61 \\
4\end{array}$ \\
\hline $\begin{array}{l}\text { Reduction in } \\
\text { DBP (mmHg) }\end{array}$ & $10.6 \pm 7.5$ & & & $12.5 \pm 4.1$ & & & $\begin{array}{l}0.24 \\
4\end{array}$ \\
\hline & Basal & 3. months & $\mathrm{p}$ & Basal & 3. months & $\mathrm{p}$ & \\
\hline Coronary & $16.84 \pm 20.9$ & $16.82 \pm 20.7$ & 0.91 & $18.47 \pm 22.4$ & $18.44 \pm 22.1$ & 0.83 & \\
\hline $\begin{array}{l}\text { narrowing as a } \\
\text { percentage } \\
(\text { mean } \pm \mathbf{S D}) *\end{array}$ & 9 & 1 & 8 & 8 & 4 & 0 & \\
\hline Coronary & $49.32 \pm 20.4$ & $48.48 \pm 20.6$ & 0.16 & $49.93 \pm 22.7$ & $49.14 \pm 22.6$ & 0.11 & \\
\hline $\begin{array}{l}\text { narrowing as a } \\
\text { percentage } \\
(\text { mean } \pm \text { SD)\# }\end{array}$ & 5 & 4 & 8 & 1 & 3 & 6 & \\
\hline
\end{tabular}

LDL, low density lipoprotein; DBP, diastolic blood pressure; SBP, systolic blood pressure; SD, standard deviation; TG; triglyceride

*non-culprit all coronary segments; \# non-culprit coronary segments, stenosis $>20 \%$ 